TRANSACTIONS OF THE

AMERICAN MATHEMATICAL SOCIETY

Volume 221, Number 2, 1976

\title{
PRESENTATIONS OF 3-MANIFOLDS ARISING FROM VECTOR FIELDS
}

\author{
BY \\ PETER PERCELL
}

\begin{abstract}
A method is given for constructing a smooth, closed, orientable 3-manifold from the information contained in a combinatorial object called an abstract intersection sequence. An abstract intersection sequence of length $n$ is just a cyclic ordering of the set $\{ \pm 1, \ldots, \pm n\}$ plus a map $v:\{1, \ldots, n\} \rightarrow\{ \pm 1\}$ It is shown that up to diffeomorphism every closed, connected, orientable 3-manifold can be constructed by the method. This is proved by showing that compact, connected, orientable 3-manifolds with boundary the 2-sphere admit vector fields of a certain type. The intersection sequences arise as descriptions of the vector fields.
\end{abstract}

1. Introduction. Several methods for constructing and presenting 3-manifolds are already well known, and the reader familiar with them will undoubtedly see relationships with our method of intersection sequences. For example, it is easy to see that from an intersection sequence presentation one can get a Heegaard diagram. However, we believe that intersection sequence presentations will prove to have some advantages over other types of presentation. It is extremely easy to enumerate all closed, orientable 3-manifolds by intersection sequence presentations, and although each manifold has infinitely many presentations, the list of presentations obtained is still in some sense short because the presentations of the fundamental groups which arise are of a quite restricted type. Only finitely many Heegaard diagrams of each genus are obtained from intersection sequence presentations because an intersection sequence of length $\boldsymbol{n}$ gives a Heegaard diagram of genus $n+1$. Furthermore, an intersection sequence presentation yields not only a manifold, but also a vector field of a certain type on the manifold, called a presentation vector field. This presentation vector field gives a richer structure to the presentations and has potential for being exploited. For example, it is known that any two presentation vector fields on a 3-ball are homotopic to each other by a homotopy consisting of a sequence of deformations from a short list of generic "basic" deformations. In [5] we will begin to

Received by the editors April 30, 1975.

AMS (MOS) subject classifications (1970). Primary 05C99, 55A05, 57A10, 57B99, 57D25; Secondary 55A20, 57D45.

Key words and phrases. Presentation, 3-manifold, intersection sequence, generic transient vector field, branched manifold. 
exploit the particular character of intersection sequence presentations by imposing the fairly natural condition of being "properly nested," and showing that it implies that the fundamental group of the presented manifold can be computed, with the computation showing that the group is a free product of cyclic groups and the manifold is a connected sum of genus one manifolds, i.e., $S^{3}, S^{2} \times S^{1}$ or lens manifolds.

In this paper we almost always deal with manifolds with boundary because the type of vector field used occurs only on manifolds with boundary. Thus intersection sequence presentations are most directly presentations of manifolds with boundary and a presentation vector field can live only on a manifold with boundary. However, since there is a one-to-one correspondence, defined by attaching or removing a 3-cell, between compact 3-manifolds with boundary $S^{2}$ and closed 3-manifolds, our work immediately yields a presentation theory for closed, orientable 3-manifolds.

2. Preliminaries. Throughout the paper, $M$ is a compact, connected $C^{\infty}$ 3-manifold with nonempty boundary $\partial M, \mathfrak{X}(M)$ is the set of $C^{\infty}$ vector fields on $M$ with the $C^{\infty}$ topology and $\mathrm{R}^{3}$ is given coordinates $(x, y, t)$, unless otherwise stated.

We recall for use in this paper some of the results of [3].

Definition. $X \in \mathfrak{X}(M)$ is called transient if each integral curve for $X$ leaves $M$ in finite positive and negative time.

Thus $X$ is transient if and only if each trajectory for $X$ is a compact arc whose endpoints are on $\partial M$. Note that although a transient vector field cannot vanish anywhere, it is possible for a trajectory to be a single point-which must lie in $\partial M$. Such a point is a point of exterior tangency in the sense that if $X$ were extended to a collar neighborhood of $M$, then the trajectory through the point would be a curve tangent to $\partial M$ from outside of $M$. The set of transient vector fields on $M$ is always nonempty and open in $\mathfrak{X}(M)$.

DEFinition. $X \in \mathfrak{X}(M)$ is called b-generic ("b" for boundary) if each $m_{0}$ $\in \partial M$ has a neighborhood $U$ for which there is an embedding $\alpha: U \rightarrow \mathbf{R}^{3}$ such that

(1) $\alpha\left(m_{0}\right)=0$,

(2) $\alpha_{*}(X \mid U)=(\partial / \partial t) \mid \alpha(U)$, where $\alpha_{*}$ is the differential map of $\alpha$, and

(3) $\alpha(U \cap \partial M)=\beta^{-1}(0) \subset \mathrm{R}^{3}$, where $\beta(x, y, t)$ is either $t, t^{2}+y$ or $t^{3}-$ $x t+y$, and $\alpha(U)$ is a "half" of $\mathbf{R}^{3}$ lying to one side of $\beta^{-1}(0)$. See Figure 1 .

The collection of b-generic vector fields is open and dense in $\mathfrak{X}(M)$. (This definition comes from Theorem 2.2 of [3] where b-generic was called condition L.)

When $X$ is a b-generic vector field on $M$, a point $m_{0} \in \partial M$ is named according to the form of the function $\beta$ associated to $m_{0}$ by the definition above. 


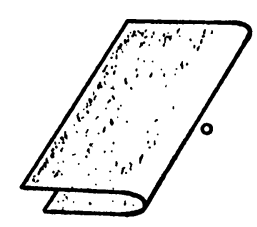

a. Tangency

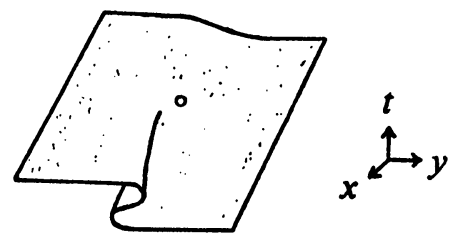

b. Inflection

\section{FIGURE 1}

(a) If $\beta(x, y, t)=t$, then $m_{0}$ is called a transverse entrance or transverse exit point according to whether $\alpha(U)$ lies above or below $\beta^{-1}(0)$ which in this case is the $x-y$ plane.

(b) If $\beta(x, y, t)=t^{2}+y$, then $m_{0}$ is called an exterior tangency or interior tangency according to whether $\alpha(U)$ lies inside $\left(t^{2}+y \leqslant 0\right.$ on $\left.\alpha(U)\right)$ or outside $\left(t^{2}+y \geqslant 0\right.$ on $\left.\alpha(U)\right)$ of the fold $\beta^{-1}(0)$.

(c) If $\beta(x, y, t)=t^{3}-x t+y$, then $m_{0}$ is called an entering inflection or exiting inflection according to whether $\alpha(U)$ lies above or below $\beta^{-1}(0)$.

The motivation for these names comes from Figure 1-remember that in these pictures the vector field, not drawn, is $\partial / \partial t$ i.e., a parallel vector field pointing up.

For $X$ a b-generic vector field, let $T$ be the set of all points in $\partial M$ where $X$ is tangent to $\partial M$, i.e., the collection of tangency and inflection points. Then $T$ is a finite union of disjointly embedded smooth circles in $\partial M$. Each of these circles separates a region of transverse entrance points from a region of transverse exit points. Furthermore, on every circle there is a finite number (possibly 0 ) of inflection points each of which separates an arc of interior tangencies from an arc of exterior tangencies. In particular, the number of inflection points on each of the circles must be even.

DEFINITION. An entering (exiting) inflection is said to point into the region of transverse entrance (exit) points partially bounded by the component of $T$ on which the inflection lies. (Again, consider Figure 1 to see the motivation for this terminology.)

DEfinition. A transient vector field $X \in \mathfrak{X}(M)$ is called generic if it is b-generic and if whenever a trajectory contains more than one point of $T$ the trajectory has a neighborhood which can be embedded in $\mathrm{R}^{3}$ so that $X$ becomes $\partial / \partial t$ and the image of the neighborhood is given by Figure 2. (The unshaded areas in the figure are the visible pieces of $\partial M$.)

The significant feature of Figure 2 is that if the two arcs of $T$ are projected along trajectories into a horizontal plane cutting through the middle of the figure, then the projected arcs have a transverse crossing at the point in the slicing plane 


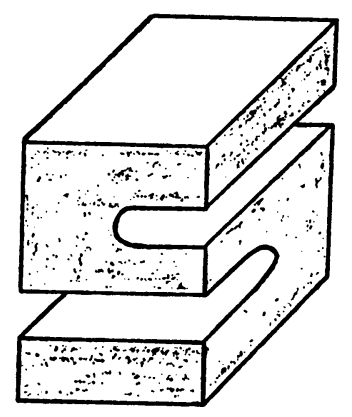

FIGURE 2

on the central multiply tangent trajectory. In particular, if $X$ is generic then a trajectory can connect no more than two interior tangencies and cannot connect an inflection to any other point in $T$. The generic transient vector fields are open and dense in the collection of all transient vector fields on $M$. In fact, they are structurally stable [3].

3. Simplifying $T$. The purpose of this section is to show that if $\partial M=S^{2}$, therr $M$ admits a generic transient vector field whose tangency set $T$ is particularly simple-just one circle of interior tangencies. This result is the foundation of our presentation theory for 3-manifolds. The result is proved by starting with an arbitrary generic vector field on $M$ and showing that it can be deformed to one of the desired type. The simplifying deformation consists of a finite sequence of "basic" deformations. Just three types of basic deformation are needed. The basic deformations all leave a vector field unchanged outside a neighborhood of an arc in $\partial M$. They are described by giving before and after pictures of the affected neighborhood embedded in $\mathrm{R}^{3}$ so that $X$ becomes $\partial / \partial t$. Only the boundary of a neighborhood is drawn, and the rest of the neighborhood can lie on either side of the drawn boundary unless otherwise stated. Each basic deformation changes a generic transient vector field into a new b-generic transient vector field which can be assumed to be fully generic since it can be made so by arbitrarily small perturbations which do not change $T$.

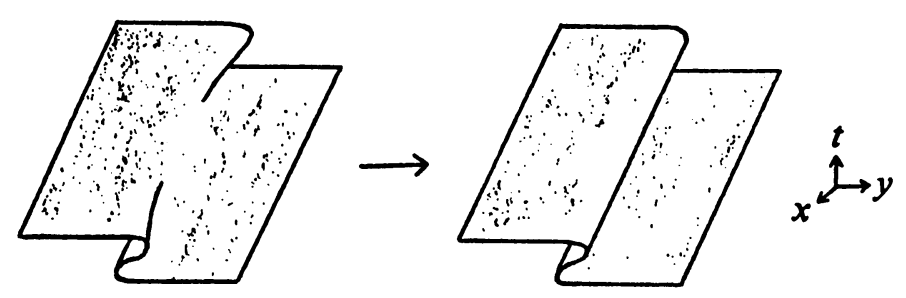

FIGURE 3. Joining Inflections 
(1) Joining Inflections. This deformation is portrayed in Figure 3. Inflections $i_{1}$ and $i_{2}$ can be joined if and only if they point into the same component, call it $C$, of $\partial M-T$ and there is a compact non-self-intersecting $\operatorname{arc} A$ such that

(a) $A \cap T=\partial A=\left\{i_{1}, i_{2}\right\}$ and $A-\partial A \subset C$,

(b) $A$ is transverse to $T$,

(c) tangent vectors to $T$ at $i_{1}$ and $i_{2}$ which point from the abutting arc of exterior tangencies to the abutting arc of interior tangencies also point to the same side of $A$. Note that only inflections of the same type (entering or exiting) can be joined by this running together operation. For those who do not quite trust pictures alone, if

$$
\beta_{0}(x, y, t)=t^{3}-\left(x^{2}+\sigma\right) t+y,
$$

then $\beta_{\sigma}^{-1}(0)$ has the before configuration when $\sigma<0$ and the after configuration when $\sigma>0$.

(2) Swallow's Tall. This deformation is portrayed in Figure 4.

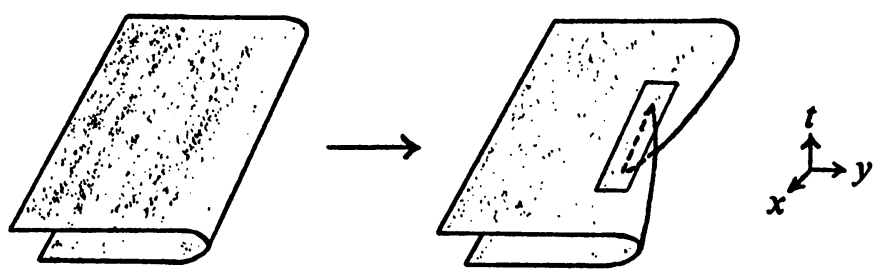

FIgURE 4. Swallow's Tail

The swallow's tail deformation can introduce a pair of inflections, one of each type, near any point on an arc of tangencies. In the after picture only one of the new inflections is visible. The other inflection is hidden underneath the fold and a new arc of tangencies which connects the inflections is hidden inside the fold. The before picture of Figure 5 shows a neighborhood in $\partial M$ of the part of $T$ hidden underneath the rectangle in Figure 4. (Note that both parts of Figure 5 represent connected sets which have been sliced through in the pictures only as an aid to understanding.) If

$$
\beta_{\sigma}(x, y, t)=\sigma\left[t^{4}-t^{2}-\epsilon x t\right]+(1-\sigma) t^{2}+y,
$$

then for $\epsilon=+1, \beta_{\sigma}^{-1}(0)$ has the before configuration of Figure 4 when $\sigma=0$ and the after configuration when $\sigma=1$. Letting $\epsilon=-1$ gives essentially the same after picture when $\sigma=1$, the only difference being a reflection across the $y$ - $t$ plane which switches the order along $T$ of the entering and exiting inflections which have been introduced.

(3) Reverse Swallow's Tall. This is the most delicate of the basic de- 


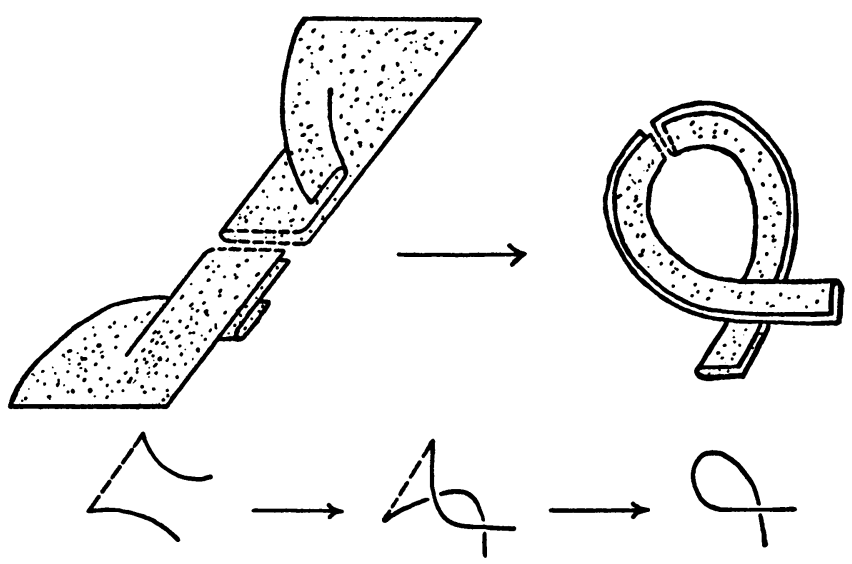

FIGURE 5

formations needed for simplifying $T$. It eliminates a pair of inflections, one of each type, if they are the boundary of an arc of exterior tangencies. If an arc of exterior tangencies $E$ has as its boundary a pair of inflections, one of each type, then there is a neighborhood $U \subset M$ of $E \cup \partial E$, an embedding $\alpha: U \rightarrow \mathbf{R}^{3}$ and a neighborhood $V \subset U \cap \partial M$ of $E \cup \partial E$ such that $\alpha_{*}(X \mid U)=(\partial / \partial t) \mid \alpha(U)$ and $\alpha(V)$ has the configuration of the before picture in Figure 5 . When this picture is being used in the context of the reverse swallow's tail deformation, $\alpha(U)$ is inside the fold connecting the inflections and extends out of the fold below the lower half and above the upper half of the figure. The deformation now takes place in two steps. The first step is modification of $X$ so that a segment of the arc of interior tangencies coming from the entering inflection is pushed over the arc of interior tangencies coming from the exiting inflection thereby creating a pair of trajectories which are twice tangent to $M$. The twice tangent trajectory nearest to the inflections is needed to get all of the after picture for the swallow's tail deformation. The second step is to exactly reverse the swallow's tail deformation thus eliminating the two inflections and the arc of exterior tangencies. The result for $\alpha(V)$ is the after picture in Figure 5-in which the image of $M$ lies outside the fold. Figure 5 also shows the effect of the two steps on $T$ alone. Note that since a trajectory of a generic transient vector field cannot connect inflection points we can assume that $V$ is small enough so that before the deformation no trajectory connects two points of $V$ which are outside of the fold. It follows that the reverse swallow's tail deformation does not destroy the property of being transient. (This is obvious for the other deformations.)

REMARK. In general it is not possible to eliminate a pair of inflections bounding an arc of interior tangencies by a reverse swallow's tail type deformation and get a transient vector field because there can be trajectories which connect 
points of an arc of interior tangencies.

Definition. $X \in \mathfrak{X}(M)$ is called simple if it is transient and generic and if $T$ is a single circle of interior tangencies.

THEOREM A. If $\partial M=S^{2}$, then $M$ admits a simple vector field.

Proof. Let $X \in \mathfrak{X}(M)$ be a generic transient vector field. If $X$ is already simple we have nothing to do, so suppose $X$ is not simple.

If $T$ is not connected, let $T_{1}$ and $T_{2}$ be distinct circles in $T$ which are both on the boundary of one of the components, call it $C$, of $\partial M-T$. Using the swallow's tail deformation and the choice of the order along $T$ of the introduced inflections it allows, we can create inflections on $T_{1}$ and $T_{2}$ which point into $C$ and which can be joined by the running together deformation. When these inflections are joined, the circles $T_{1}$ and $T_{2}$ are connected to become one circle. Thus by applying a sequence of swallow's tail and joining deformations to $X$, we can make $T$ connected, i.e., a single circle.

At this stage the modified $X$ cannot be simple because the process for connecting $T$ must have left inflections on $T$ if $T$ was not already connected. Therefore $T$ is either one circle with inflections on it or one circle of exterior tangencies (in which case $M=D^{3}$ ). In the latter case, the swallow's tail deformation can be used to introduce inflections so we can assume that $T$ is a circle with inflections. It now follows from the assumption that $\partial M=S^{2}$ and a straightforward application of Pugh's generalized Poincare index formula [6] that the number of entering inflections is the same as the number of exiting inflections.

All we have left to do is to eliminate the inflections. As long as inflections remain on $T$ either the reverse swallow's tail deformation can be used to cancel a pair of inflections or we can proceed as follows. If the reverse swallow's tail cannot be used, then there is an arc of exterior tangencies whose endpoints are a pair of inflections of the same type. Denote the type (entrance or exit) of these two inflections by $\tau$ and the other type by $-\tau$. It is easy to see that the two inflections can be run together. When this is done, $T$ breaks into two components $T_{1}$ and $T_{2}$ which bound an annulus of transverse points of type $-\tau$ and one of the components, say $T_{1}$, is a circle of exterior tangencies. Furthermore, $T_{2}$ must contain two more inflections of type $-\tau$ than of type $\tau$, so there is an arc of tangency points in $T_{2}$ whose endpoints are both inflections of type $-\tau$ which point into the annulus. Thus if we use the swallow's tail deformation to introduce a pair of inflections on $T_{1}$, then the new inflection of type $-\tau$ on $T_{1}$ points into the annulus and can be joined with an inflection already on $T_{2}$. This reconnects $T_{1}$ and $T_{2}$ and the net effect has been to reduce the number of inflection points in $T$ by two.

Note that with this procedure the last pair of inflections is always eliminated 
by the reverse swallow's tail deformation so in the end $T$ is a circle of interior, not exterior, tangencies.

4. The space $M / X$. In this section we introduce the concept of a normal, branched 2-manifold because if $X$ is a simple vector field on $M$, then the quotient space $M / X$ obtained by collapsing trajectories of $X$ to points has this useful structure.

The concept of branched manifolds is due to R. F. Williams-see [8] and [9]. In this paper we only need a very simple class of branched 2-manifolds which we now describe. Let $\phi: \mathbf{R} \rightarrow \mathbf{R}$ be a fixed $C^{\infty}$ function such that $\phi(s)=$ 0 for $s \leqslant 0$ and $\phi(s)>0$ for $s>0$, let

$$
\begin{aligned}
& P_{1}=\left\{(x, y, t) \in \mathbf{R}^{3} \mid t=0\right\}, \\
& P_{2}=\left\{(x, y, t) \in \mathbf{R}^{3} \mid t=-\phi(x)\right\}, \\
& P_{3}=\left\{(x, y, t) \in \mathbf{R}^{3} \mid t=\phi(y)\right\},
\end{aligned}
$$

let $U_{1}=P_{1}, U_{2}=P_{1} \cup P_{2}$ and $U_{3}=P_{1} \cup P_{2} \cup P_{3}$, and let $p: U_{k} \rightarrow \mathrm{R}^{2}, k=$ $1,2,3$, by $p(x, y, t)=(x, y)$. Differentiability for maps whose domain or range is $U_{2}$ or $U_{3}$ is defined as follows. Suppose $N$ is an arbitrary manifold, $k, l=2$ or 3 and $V_{k}$ is an open subset of $U_{k}$.

(a) $f: N \rightarrow U_{k}$ is called smooth if $p \circ f$ is smooth.

(b) $f: V_{k} \rightarrow N$ is called smooth if $f \mid\left(P_{i} \cap V_{k}\right)$ is smooth for $1 \leqslant i \leqslant k$.

(c) $f: V_{k} \rightarrow U_{l}$ is called smooth if $p \circ\left(f \mid\left(P_{i} \cap V_{k}\right)\right)$ is smooth for $1 \leqslant$ $i \leqslant k$.

The sets $U_{1}, U_{2}$ and $U_{3}$ are used as local models for a normal, branched 2-manifold the same way that $U_{1}$ alone is used as the local model for a closed 2-manifold.

Definitions. A (smooth) normal, branched 2-manifold is a compact Hausdorff space $Q$ which has a covering by open sets $V_{1}, V_{2}, \ldots, V_{n}$ such that

(1) for each $i=1, \ldots, n$, there is a $k(i) \in\{1,2,3\}$ such that there is a homeomorphism $\alpha_{i}: V_{i} \rightarrow U_{k(i)}$, and

(2) for $i, j=1, \ldots, n, \alpha_{i} \circ \alpha_{j}^{-1}: \alpha_{j}\left(V_{i} \cap V_{j}\right) \rightarrow U_{k(i)}$ is smooth. The branch set of $Q$, denoted $B(Q)$, is the set of points which correspond to a point of the $y$-axis in $U_{2}$ or a point of the $x$-axis or $y$-axis in $U_{3}$. The set of crossing points, denoted $C(Q)$, is the (finite) set of points corresponding to the origin in $U_{3}$.

Note that a normal. branched 2-manifold $Q$ has a tangent bundle $T Q$ because the planes $P_{1}, P_{2}$ and $P_{3}$ lie flat against each other at points of intersection. Smoothness of maps whose domain or range is a normal, branched 2-manifold is defined using the coordinate systems $\left(V_{i}, \alpha_{i}\right)$ in the same way that smoothness is defined for maps of ordinary manifolds and smooth maps induce differential maps of tangent bundles as usual.

Definition. A smooth map $f: S^{1} \rightarrow Q$, where $S^{1}$ is the circle and $Q$ is a 
normal, branched 2-manifold, is called a normal immersion if

(a) $f$ is an immersion, i.e., $f_{*}(v) \neq 0$ for all nonzero $v \in T S^{1}$, where $f_{*}$ is the differential map induced by $f$,

(b) $f^{-1}(y)$ contains at most 2 points for every $y \in Q$, and

(c) if $f^{-1}(y)$ contains two distinct points $x_{1}$ and $x_{2}$, then $f_{*}\left(v_{1}\right)$ and $f_{*}\left(v_{2}\right)$ are linearly independent whenever $v_{1}$ and $v_{2}$ are nonzero tangent vectors to $S^{1}$ at $x_{1}$ and $x_{2}$ respectively.

Proposition 1. Let $X$ be a simple vector field on $M$, let $T$ be its circle of tangency points and let $\pi: M \rightarrow M / X$ be the projection map.

(1) The quotient space $M / X$ is a normal, branched 2-manifold.

(2) $\pi \mid T$ is a normal immersion whose image is $B(M / X)$.

(3) $B(M / X)$ always has an orientable neighborhood.

(4) $M / X$ is orientable if and only if $M$ is orientable.

(5) $M$ and $M / X$ have isomorphic fundamental groups.

Proof. (1) and (2) are obvious-see Figure 6-and (5) follows from a
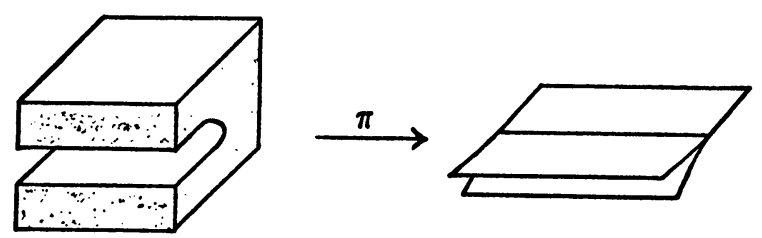

a. Branching
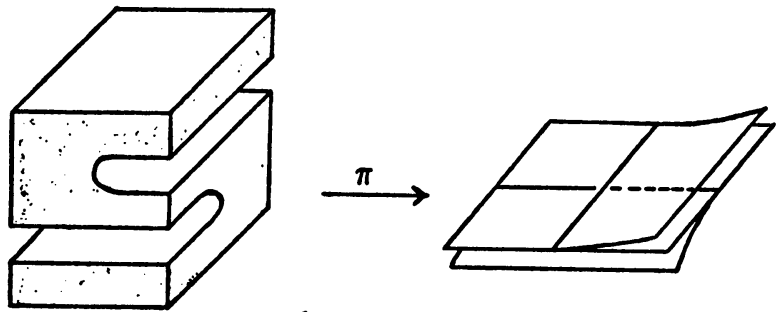

b. Crossing

FIGURE 6

result of Smale [7]. Suppose $T$ is oriented by a nonvanishing tangent vector field $\theta$ and $\eta$ is a vector field along $T$ which is transverse to $\partial M$ and points into $M$. Then $\theta$ and $\eta$ extend to vector fields, denoted by the same letters, on a small neighborhood $U$ of $T$ in $M$ such that $\{\theta(m), \eta(m), X(m)\}$ and $\left\{\pi_{*}(\theta(m))\right.$, $\left.\pi_{*}(\eta(m))\right\}$ are linearly independent sets for each $m \in U$. Thus the ordered triple $(\theta, \eta, X)$ can be taken to define the positive orientation for $U$ and the ordered pair $\left(\pi_{*}(\theta), \pi_{*}(\eta)\right)$ can be used to define the positive orientation for $\pi(U)$, which is a neighborhood of $B(M / X)$. Note that if $y=\pi\left(m_{1}\right)=\pi\left(m_{2}\right)$, then $\left(\pi_{*}\left(\theta\left(m_{i}\right)\right)\right.$, $\left.\pi_{*}\left(\eta\left(m_{i}\right)\right)\right), i=1,2$, define the same orientation of $T_{y}(M / X)$. This proves (3). 
After this argument it is easy to see how to prove (4).

5. Intersection sequences and tubular neighborhoods. An intersection sequence is a combinatorial object usually associated with a normal immersion of an oriented circle into an oriented 2-manifold, see [4] for example. In this section we review this idea while adapting it to present needs. We will continue to use the notation of Proposition 1 and its proof.

DefinItion. An abstract intersection sequence, denoted IS, is a triple $(n, \sigma, v)$ consisting of

(a) a nonnegative integer $n$,

(b) a cyclic ordering of $I_{n}=\{ \pm 1, \ldots, \pm n\}$ given by a bijection $\sigma: I_{n}$ $\rightarrow I_{n}$ which acts transitively on $I_{n}$, i.e., for every $i, j \in I_{n}$ there is a positive integer $k$ such that $\sigma^{k}(i)=j$ (where $\sigma^{k}$ is composition of $\sigma$ with itself $k$ times), and

(c) a map $v: J_{n} \rightarrow\{ \pm 1\}$, where $J_{n}=\{1, \ldots, n\}$.

Note that if $n=0$, then $\sigma$ and $\nu$ are the empty mapping with empty domain.

Definition. Suppose $C(M / X)$ has $n$ points. A labeling $L$ of $X$ is a naming of the points of $C(M / X)$ as $y_{1}, \ldots, y_{n}$ and a naming of their preimages in $T$ by

$$
(\pi \mid T)^{-1}\left(y_{j}\right)=\left\{x_{-j}, x_{j}\right\}, \quad j \in J_{n},
$$

where $x_{-j}$ is below or before $x_{j}$ along the oriented $X$-trajectory $\pi^{-1}\left(y_{j}\right)$.

Definition. Given a labeling $L$ of a simple vector field $X$ on $M$ and a vector field $\theta$ which orients $T$, the intersection sequence $\operatorname{IS}(M, X, L, \theta)$ is defined to be the abstract intersection sequence $(n, \sigma, \nu)$ determined as follows:

(a) $n$ is the number of points in $C(M / X)$,

(b) for each $i \in I_{n}, \sigma(i)$ is the $k \in I_{n}$ such that $x_{k}$ is the first $x_{l}$ encountered when moving away from $x_{i}$ along $T$ in the positive direction, and

(c) for $j \in J_{n}, \nu(j)$ is the orientation of the crossing at $y_{j}$ which is defined to be +1 or -1 according to whether the ordered pair $\left(\pi_{*}\left(\theta\left(x_{-j}\right)\right), \pi_{*}\left(\theta\left(x_{j}\right)\right)\right)$ in $T_{y_{j}}(M / X)$ is positively or negatively oriented (where the orientation of a neighborhood of $B(M / X)$ is determined by $\theta$ as in the proof of Proposition 1).

Note that $\operatorname{IS}(M, X, L, \theta)$ is an intersection sequence in the sense of [4] for the normal immersion $\pi \mid T$.

We now show how to use the information contained in an abstract intersection sequence IS to construct a space $N(I S)$ which acts as a tubular neighborhood for the immersed circle $B(M / X)$ whenever IS is an intersection sequence for a simple vector field $X$ on $M$. Let

$$
A_{e}=\left\{(r, \theta, z) \in \mathbf{R}^{3}:|r-1| \leqslant e \text { and } z= \pm \phi(r-1)\right\}
$$

where $0<e<1,(r, \theta, z)$ are cylindrical coordinates on $\mathbf{R}^{3}$ and $\phi$ is the function 
used to define the local models for normal, branched 2-manifolds. $A_{e}$ is a branched annulus whose branching is to the outside of the unit circle, which is the branch set. $A_{e}$ is oriented by letting the pair $(\partial / \partial r, \partial / \partial \theta)$ be positively oriented. Suppose IS $=(n, \sigma, v)$. For $k=0,1, \ldots, 2 n-1$, label the point $(1, \pi(k+1) / n, 0)$ with the name $x_{\sigma^{k}}(1)$. Then the points $x_{ \pm 1}, \ldots, x_{ \pm n}$ are distributed uniformly around the unit circle in $A_{e}$ in the cyclic order given by $\sigma$. For $i \in I_{n}$, let $\theta_{i}$ be the $\theta$ coordinate of $x_{i}$ and let $e$ be small enough so that the "branched squares"

$$
S_{i}=\left\{(r, \theta, z) \in A_{e}:|r-1| \leqslant e,\left|\theta-\theta_{i}\right| \leqslant e \text { and } z= \pm \phi(r-1)\right\}
$$

are pairwise disjoint. Let $N(\mathrm{IS})$ be the space obtained from $A_{e}$ by identifying the top of the square $S_{-j}$ with the bottom of the square $S_{j}$, where $j \in J_{n}$, according to the rule

$$
\left(1+s, \theta_{-j}+t, \phi(s)\right) \sim\left(1+\nu(j) t, \theta_{j}-v(j) s,-\phi(\nu(j) t)\right) .
$$

Thus, according to whether $v(j)=+1$ or -1 the square $S_{j}$ is rotated counterclockwise or clockwise through $90^{\circ}$ before the identification is made. It is easily seen that $N(\mathrm{IS})$ is an orientable, normal, branched 2-manifold with boundary and that the boundary is a finite collection of piecewise smooth circles. $N(I S)$ has been defined so that we have the following

PROPOSITION 2. Let IS be an intersection sequence for a simple vector field $X$ on $M$. Then $B(M / X)$ has a neighborhood $\widetilde{N}(\mathrm{IS})$ which is diffeomorphic to $N($ IS).

6. The presentation theory. In this section we show how abstract intersection sequences act as presentations of orientable 3-manifolds by showing how to construct a manifold $M$ (IS) from an abstract intersection sequence IS and by showing that included among the manifolds which can be constructed this way are all orientable $M$ such that $\partial M=S^{2}$. Unless otherwise stated, notation used is that of the last two sections.

Definition. A vector field $X$ on $M$ is called a presentation vector field if $X$ is simple and $M / X-B(M / X)$ is a disjoint union of open 2-disks.

Note that if $M$ admits a presentation vector field, then $M$ is orientable since $M / X$ is orientable because it is obtained by capping off the boundary circles of the orientable space $\widetilde{N}(\mathrm{IS})$ of Proposition 2 by 2-disks.

Definition. For $i=1,2$, let $X_{i} \in \mathfrak{X}\left(M_{i}\right)$. The pairs $\left(M_{1}, X_{1}\right)$ and $\left(M_{2}\right.$, $X_{2}$ ) are called equivalent if there is a diffeomorphism $\alpha: M_{1} \rightarrow M_{2}$ which takes trajectories of $X_{1}$ to trajectories of $X_{2}$ and is sense preserving on trajectories.

THEOREM B. For every abstract intersection sequence IS, there is a pair (M(IS), X(IS)) consisting of an orientable, compact, connected, $C^{\infty} 3$-manifold 
with boundary and a presentation vector field on the manifold such that IS is an intersection sequence for $X(\mathrm{IS})$. Furthermore, the pair (M(IS), $X(\mathrm{IS}))$ is unique up to equivalence.

PRoof. Let $Q($ IS) be the smooth, orientable, normal, branched 2-manifold obtained by forming $N$ (IS) and capping off its boundary circles with 2-disks. $Q(I S)$ is "thickened" to form the manifold $M$ (IS) with a foliation whose leaves are compact intervals by thickening $N$ (IS) in the obvious way (locally this amounts to going backwards in Figure 6) and then thickening the complementary 2-disks in $Q$ (IS) by forming the cartesian product with a compact interval. Since $N$ (IS) has well-defined upper and lower branches at each branch point, the foliation of $M(I S)$ can be oriented so that the leaves are directed "up". Thus there is a vector field $X($ IS) whose directed trajectories are the oriented foliation of $M($ IS). It is clear from the construction that $X(\mathrm{IS})$ is a presentation vector field and that IS is an intersection sequence for $X$ (IS).

If $(M, X)$ is any other pair such that $X$ is a presentation vector field on $M$ and IS is an intersection sequence for $X$, then we construct a diffeomorphism giving an equivalence with ( $M(\mathrm{IS}), X(\mathrm{IS}))$ by first defining it between saturated (with respect to trajectories) neighborhoods of the tangency sets using Proposition 2 and then extending it into the remainder of the manifolds.

THEOREM C. Suppose $\partial M=S^{2}$ and $M$ is orientable. Then $M$ admits a presentation vector field. Hence $M$ can be presented by an abstract intersection sequence, i.e., there exists an IS such that $M$ is diffeomorphic to $M$ (IS).

Proof. By Theorem A, $M$ admits simple vector fields. Let $X$ be a simple vector field on $M$, let IS be an intersection sequence for $X$ and let $\widetilde{N}(\mathrm{IS})$ be a neighborhood of $B(M / X)$ which is diffeomorphic to $N(I S)$. Define the deficiency of $X$, denoted $d(X)$, to be the number of boundary circles of $N($ IS) minus the number of components of $M / X-\widetilde{N}(\mathrm{IS})$. Note that $d(X) \geqslant 0$ because the closure of each component of $M / X-\widetilde{N}(\mathrm{IS})$ has a nonempty boundary which is a collection of boundary circles of $\widetilde{N}($ IS). We will prove by induction on $d(X)$ that $X$ can be deformed to a presentation vector field.

Let $C$ be a component of the closure of $M / X-\widetilde{N}($ IS). Then $C$ is a compact 2-manifold without branching whose boundary is a set of boundary circles of $\widetilde{N}(I S)$. Also, $\pi^{-1}(C) \cap \partial M$ has two components each of which is a submanifold of $\partial M=S^{2}$ diffeomorphic to $C$, so $C$ is either a disk or a disk with holes. If $d(X)=0$, then $C$ cannot have more than one boundary circle, so it must be a disk. Thus if $d(X)=0$, then $X$ is already a presentation vector field.

To finish the proof by induction we show that if $d(X)>0$, then $X$ can be deformed to a new simple vector field $X^{\prime}$ such that $d\left(X^{\prime}\right)=d(X)-1$. When 
$d(X)>0, C$ can be chosen so that it has at least two boundary circles. Let $\widetilde{C}$ be the component of $M / X-B(M / X)$ which contains $C$. Choose $b_{1}$ and $b_{2}$ to be distinct points in $B(M / X)-C(M / X)$ which are in the closure of different components of $\widetilde{C}-C$. Then there is a compact non-self-intersecting $\operatorname{arc} A$ in $M / X$ such that

(a) $A$ is transverse to $B(M / X)$ and $\partial C$,

(b) $A \cap B(M / X)=\partial A=\left\{b_{1}, b_{2}\right\}$ and $A-\partial A \subset \widetilde{C}$,

(c) $A \cap \partial C$ consists of exactly two points which lie on different circles of $\partial C$.

Thus $A$ is divided into three segments so that the middle segment lies in $C$ and the two end segments lie in different components of $\widetilde{C}-C$.

Since $b_{1}$ and $b_{2}$ are distinct branch points which are not crossing points, they have disjoint compact neighborhoods $V_{1}$ and $V_{2}$ which are both diffeomorphic to the branched square of Figure 6a. Then $V_{i}-B(M / X) \cap V_{i}$ consists of three branches, which can unambiguously be called inner, upper and lower branches if we say that $X$ points "up". It is clear that $V_{1}$ and $V_{2}$ can be chosen so that they are neighborhoods in $\widetilde{N}(\mathrm{IS})$ of the two end segments of $A$ and so that the edges of $\partial V_{1}$ and $\partial V_{2}$ which are parallel to $B(M / X)$ lie in $\partial \tilde{N}(I S)$. Note that the end of $A$ at $b_{i}$ meets just one of the branches of $V_{i}$, so each end of $A$ is uniquely in either an inner, upper or lower branch.

We now claim that we can assume that $b_{1}, b_{2}$ and $A$ were chosen so that the ends of $A$ are not both in upper branches or both in lower branches. This follows from the easily checked fact that except when $B(M / X)$ has no crossing points at all, no boundary circle of $N(I S)$ can consist of segments all in upper branches of $N(I S)$ or all in lower branches of $N(I S)$. (Actually, with a slightly longer argument, it can be shown that for any permissible choice of $b_{1}, b_{2}$ and $A$ the ends of $A$ are not both in upper or both in lower branches.) Thus there are four possibilities for the branches containing the ends of $A$ : inner and inner, inner and upper, inner and lower or upper and lower.

In any case, $A$ has a compact neighborhood $V$ which is the union of $V_{1}$, $V_{2}$ and a strip $S$ in $C$ which is a tubular neighborhood of $A \cap C$ meeting $\partial C$ in the same two arcs as $V_{1}$ and $V_{2}$. Then $V$ can be embedded in $\mathrm{R}^{3}$ so that the branched squares $V_{1}$ and $V_{2}$ are transverse to the vertical direction and so that the upper branch lies above the lower branch in the images of $V_{1}$ and $V_{2}$. Furthermore, since $M$ is orientable, the embedding of $V$ can be chosen so that the strip $S$ is also transverse to the vertical direction. The conditions on the embedding of $V$ ensure that a slight vertical thickening of the image of $V$ gives an em. bedding of $\pi^{-1}(V)$ in $\mathbf{R}^{3}$ such that $X \mid \pi^{-1}(V)$ becomes $\partial / \partial t$ on the image of $\pi^{-1}(V)$.

It should now be easy to see that in all the cases there is a deformation of 
$X$ which is supported in $\pi^{-1}(V)$ and which has the effect on $M / X$ of pushing a segment of one of the arcs of $B(M / X) \cap V$ along $A$ and then over the other arc of $B(M / X) \cap V$ in such a way that two new transverse crossing points and a new component, which is a disk, of $M / X-B(M / X)$ are created. Before and after pictures of the deformation as seen through its effect on $M / X$ are given in Figure 7 for one of the cases. The new vector field, call it $X^{\prime}$, is obviously simple.

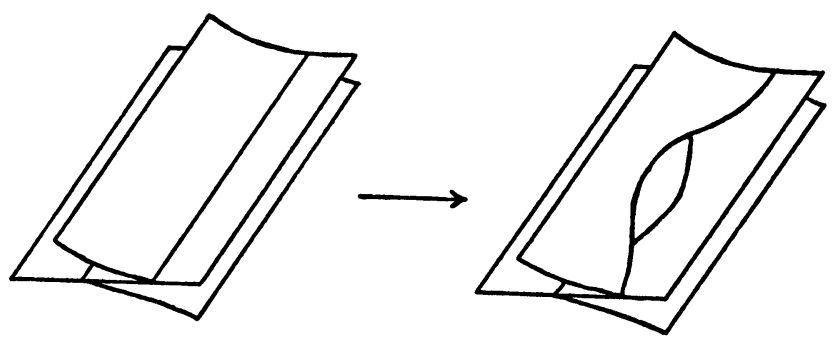

FIGURE 7

Finally, $d\left(X^{\prime}\right)=d(X)-1$ because if IS' is an intersection sequence for $X^{\prime}$, then $M / X^{\prime}-\widetilde{N}\left(I^{\prime}\right)$ has one more component, a new disk, than $M / X-\widetilde{N}($ IS $)$, but $N\left(\mathrm{IS}^{\prime}\right)$ has the same number of boundary circles as $N(\mathrm{IS})$, since two of the boundary circles of $C$ are connected by the deformation in such a way that they give one boundary circle for the component of $M / X^{\prime}-\tilde{N}\left(\mathrm{IS}^{\prime}\right)$ corresponding to $C$, while only the single boundary circle for the new disk is created.

The last statement of the theorem now follows because if $X$ is a presentation vector field on $M$ and IS is an intersection sequence for $X$, then $M$ is diffeomorphic to $M(\mathrm{IS})$ by the uniqueness result in Theorem B.

In order to better understand the problems this proof deals with, the reader is urged to discover and consider the (unique) pair $(M, X)$ such that $\partial M=S^{2}$, $M$ is orientable and $X$ is a simple vector field on $M$ for which $(0, \phi, \phi)$ is an intersection sequence, where $\phi$ denotes the empty mapping.

COROLLARY. Every closed, orientable 3-manifold can be presented by (constructed from) an abstract intersection sequence.

7. The boundary of $M(I S)$. Since those intersection sequences IS for which $\partial M($ IS $)=S^{2}$ present all closed, orientable 3-manifolds, but in general $\partial M($ IS $)$ need not be $S^{2}$, we give a criterion which can be used effectively to pick out the IS such that $\partial M($ IS $)=S^{2}$. What we do is basically quite similar to Neuwirth's work [1]. The last result of the paper is a condition which the fundamental group of a closed, orientable 3-manifold must satisfy.

Let $X$ be a simple vector field on $M$. Then $\partial M$ is separated by the tangency circle $T$ into two open regions. One, denoted $R_{e}$, is the region of transverse entrance points and the other, denoted $R_{x}$, is the region of transverse exit points. $T$ is the boundary of each of these regions. 
Definition. Let $X$ be a simple vector field on $M$ with tangency circle $T$. The entrance graph, denoted $G_{e}$, is defined by

$$
G_{e}=\pi^{-1}(\pi(T)) \cap \bar{R}_{e},
$$

where $\pi: M \rightarrow M / X$ is the projection map and the bar denotes closure. Similarly, the exit graph, denoted $G_{x}$, is defined by

$$
G_{x}=\pi^{-1}(\pi(T)) \cap \bar{R}_{x} .
$$

Thus the entrance and exit graphs are the projections of $T$ along trajectories into the closures of the entrance and exit regions.

THEOREM D. Let IS $=(n, \sigma, v)$ be an abstract intersection sequence. Then the boundary of $M(\mathrm{IS})$ is the 2-sphere if and only if

(a) the entrance graph $G_{e}$ and the exit graph $G_{x}$ for $X(\mathrm{IS})$ are connected, and

(b) $N$ (IS) has $n+1$ boundary circles.

Proof. Clearly $\partial M($ IS $)=S^{2}$ if and only if $\bar{R}_{e}$ and $\bar{R}_{x}$ are closed 2-disks, where $R_{e}$ is the entrance region and $R_{x}$ is the exit region for $X(\mathrm{IS})$. Since $\bar{R}_{e}$ and $\bar{R}_{x}$ are both 2-manifolds whose boundary is the circle $T$ of tangency points for $X(I S)$, they are 2-disks if and only if they are connected and have Euler characteristic equal to 1 .

Let $Q=M(\mathrm{IS}) / X(\mathrm{IS})$ and let $\pi: M(\mathrm{IS}) \rightarrow Q$ be the projection map. Then $Q-B(Q)$ is a disjoint union of exactly as many open 2-disks as there are boundary circles in $N(\mathrm{IS})$. Call the disks $D_{1}, \ldots, D_{K}$ and for $i=1, \ldots, k$, let

$$
D_{i}^{e}=\pi^{-1}\left(D_{i}\right) \cap R_{e} \text { and } D_{i}^{x}=\pi^{-1}\left(D_{i}\right) \cap R_{x} \text {. }
$$

It follows that $\bar{R}_{e}$ is just $G_{e}$ with the 2-cells $D_{1}^{e}, \ldots, D_{k}^{e}$ attached and $\bar{R}_{x}$ is $G_{x}$ with the 2-cells $D_{1}^{x}, \ldots, D_{k}^{x}$ attached. Clearly connectedness for $\bar{R}_{e}$ and $\bar{R}_{x}$ is equivalent to connectedness for $G_{e}$ and $G_{x}$. Thus to finish the proof we only need to show that $\bar{R}_{e}$ and $\bar{R}_{x}$ have Euler characteristic 1 if and only if $k=n+1$. Consideration of Figure $6 \mathrm{~b}$ shows easily that $G_{e}$ is a graph, i.e., one dimensional cell complex, such that the number $v$ of vertices is $2 n$ and each vertex is the endpoint of exactly 3 edges-for each crossing point in $Q$ there is one vertex of $G_{e}$ on $T$ and one vertex in $R_{e}$. Therefore the number $e$ of edges for $G_{e}$ is $3 n$, so the Euler characteristic of $\bar{R}_{e}$ is

$$
v-e+k=2 n-3 n+k=k-n \text {. }
$$

Hence the Euler characteristic of $\bar{R}_{e}$ is 1 if and only if $k=n+1$. A similar argument shows that the Euler characteristic of $\bar{R}_{x}$ is 1 if and only if $k=n+1$.

Although the details are somewhat tedious, putting this criterion into the 
form of an algorithm is fairly straightforward. In fact, the author has written a computer program whose output is a list of those intersection sequences IS of a given length such that $\partial M(I S)=S^{2}$ together with the boundary circles of $N($ IS). These boundary circles give the relations for a presentation of the fundamental group of $M$ (IS).

Our final result is a slight strengthening of a result of Neuwirth [2] which falls out naturally at this point.

COROLlARY. The fundamental group of a closed, orientable 3-manifold $\widetilde{M}$ has a finite presentation with an equal number of generators and relations such that each generator appears exactly 3 times in the relations.

Proof. $\widetilde{M}$ can be constructed from an intersection sequence IS $=(n, \sigma, \nu)$ such that $\partial M(\mathrm{IS})=S^{2}$. Then $\widetilde{M}, M(\mathrm{IS})$ and $Q=M(\mathrm{IS}) / X(\mathrm{IS})$ all have the same fundamental group. A presentation for the fundamental group of $Q$ is obtained by picking a maximal tree in the graph $B(Q)$ and letting the generators correspond to the $n+1$ edges not in the maximal tree. Since the relations correspond to the $n+1$ boundary circles of $N($ IS), each generator appears exactly 3 times in the relations because the branched strip in $N$ (IS) which follows an edge of the graph $B(Q)$ has exactly 3 arcs in its boundary.

8. Addendum. Shortly after writing this paper I became aware of Wilson's work [10] and realized that a considerably shorter and more elementary proof of Theorem $A$ than the one in $\$ 3$ can be given. However, I want to leave that proof in the paper as it stands because the techniques used seem to be of independent interest and because the original proof actually shows more than the theorem states while the new proof does not yield the same additional information. It follows immediately from the original proof that regardless of what the boundary of $M$ is, every b-generic vector field on $M$ (not necessarily transient) is homotopic to a b-generic vector field which has at most one circle of its tangency set $T$ on each component of $\partial M$ and all inflections of the same type on each circle of $T$. The homotopy can be obtained by extending the vector field into a collar neighborhood of $M$, then leaving the vector field fixed while suitably deforming $\partial M$, and finally pulling back to the original $M$ to get a deformation of the vector field rather than the manifold. Thus the homotopy can be taken to be fixed away from a small neighborhood of $\partial M$.

The following is a sketch of the new proof of Theorem A. Let $\widetilde{M}$ be the closed 3-manifold gotten from $M$ by capping off its boundary with a 3-cell. Then $\widetilde{M}$ admits a nonsingular vector field, call it $\widetilde{X}$. Wilson [10] showed that a finite number of disjoint flow boxes (closed sets in $\widetilde{M}$ diffeomorphic to the product of a 2-disk and an interval so that the family of intervals corresponds to segments of trajectories of $\widetilde{X}$ ) can be found such that the trajectory through any 
point enters a flow box in both positive and negative time. However, a single flow box is all that is really needed because it is not hard to show that the finitely many flow boxes can be connected by tubes which are themselves flow boxes in such a way that a single "large" flow box is formed. If we now inscribe a 2-sphere in this flow box (which is diffeomorphic to a cylinder) and remove the interior of the sphere from $\widetilde{M}$, then what remains is clearly $M$ with a b-generic transient vector field on it whose tangency set is a single circle of interior tangencies. This vector field can be made generic and hence simple by arbitrarily small perturbations.

\section{REFERENCES}

1. L. Neuwirth, An algorithm for the construction of 3-manifolds from 2-complexes, Proc. Cambridge Philos. Soc. 64 (1968), 603-613. MR 37 \#231.

2. Some algebras for 3-manifolds, Topology of Manifolds (Proc. Inst., Univ. of Georgia, Athens, Ga., 1969), Markham, Chicago, Ill., 1970, pp. 179-184. MR 43 \#2716.

3. P. Percell, Structural stability on manifolds with boundary, Topology 12 (1973), 123-144. MR 48 \#1267.

4. - The genus of an abstract intersection sequence, Proc. Amer. Math. Soc. (to appear).

5. - Properly nested presentations of 3-manifolds (to appear).

6. C. Pugh, A generalized Poincaré index formula, Topology 7 (1968), 217-226. MR 37 \#4828.

7. S. Smale, $A$ Vietoris mapping theorem for homotopy, Proc. Amer. Math. Soc. 8 (1957), 604-610. MR 19, 302.

8. R. F. Williams, One dimensional non-wandering sets, Topology 6 (1967), 473-487. MR 36 \#897.

9. - Expanding attractors, Inst. Hautes Etudes Sci. Publ. Math. No. 43 (1974), 169-203. MR 50 \#1289.

10. F. W. Wilson, Jr., On the minimal sets of non-singular vector fields, Ann. of Math. (2) 84 (1966), 529-536. MR 34 \#2028.

DEPARTMENT OF MATHEMATICS, UNIVERSITY OF CHICAGO, CHICAGO, ILLINOIS 60637 77004

Current address: Department of Mathematics, University of Houston, Houston, Texas 